\title{
Predicting Conceptual Processing Capacity from Spontaneous Neuronal Activity of the Left Middle Temporal Gyrus
}

\author{
Tao Wei, ${ }^{1,2}$ Xia Liang, ${ }^{1}$ Yong He, ${ }^{1}$ Yufeng Zang, ${ }^{1}$ Zaizhu Han, ${ }^{1}$ Alfonso Caramazza,,${ }^{3,4}$ and Yanchao $\mathrm{Bi}^{1}$ \\ ${ }^{1}$ State Key Laboratory of Cognitive Neuroscience and Learning, Beijing Normal University, Beijing 100875, China, ${ }^{2}$ Department of Psychology, Rice \\ University, Houston, Texas 77251, ${ }^{3}$ Department of Psychology, Harvard University, Cambridge, Massachusetts 02138, and ${ }^{4}$ Center for Mind/Brain Sciences, \\ University of Trento, I-38122 Trento, Italy
}

Conceptual processing is a crucial brain function for humans. Past research using neuropsychological and task-based functional brainimaging paradigms indicates that widely distributed brain regions are related to conceptual processing. Here, we explore the potential contribution of intrinsic or spontaneous brain activity to conceptual processing by examining whether resting-state functional magnetic resonance imaging (rs-fMRI) signals can account for individual differences in the conceptual processing efficiencies of healthy individuals. We acquired rs-fMRI and behavioral data on object conceptual processing tasks. We found that the regional amplitude of spontaneous low-frequency fluctuations in the blood oxygen level-dependent signal in the left (posterior) middle temporal gyrus (LMTG) was highly correlated with participants' semantic processing efficiency. Furthermore, the strength of the functional connectivity between the LMTG and a series of brain regions-the left inferior frontal gyrus, bilateral anterior temporal lobe, bilateral medial temporal lobe, posterior cingulate gyrus, and ventromedial and dorsomedial prefrontal cortices-also significantly predicted conceptual behavior. The regional amplitude of low-frequency fluctuations and functionally relevant connectivity strengths of LMTG together accounted for 74\% of individual variance in object conceptual performance. This semantic network, with the LMTG as its core component, largely overlaps with the regions reported in previous conceptual/semantic task-based fMRI studies. We conclude that the intrinsic or spontaneous activity of the human brain reflects the processing efficiency of the semantic system.

\section{Introduction}

Semantic memory is a system for the storage, retention, and recall of general conceptual knowledge about objects, people, facts, and beliefs that are unrelated to specific experiences (Tulving, 1972). This system serves as a foundation for various cognitive processes including language, object recognition and use, reasoning, and problem solving. Evidence from neuropsychological and task-based functional brain-imaging studies has shown that performing semantic/ conceptual tasks (we do not intend to distinguish between semantics and conceptual knowledge here, and use these two terms interchangeably) implicates multiple areas in the temporal, frontal, and frontoparietal regions (Dronkers et al., 2004; Binder et al., 2009).

To identify how conceptual knowledge is maintained at rest in the absence of specific inputs or outputs, the current study explores the role of spontaneous brain activity in semantic memory

Received April 19, 2011; revised Nov. 2, 2011; accepted Nov. 5, 2011.

Author contributions: T.W., Y.H., and Y.B. designed research; T.W. performed research; T.W., X.L., Y.H., Y.Z., and A.C. analyzed data; T.W., Z.H., A.C., and Y.B. wrote the paper.

This work was supported by the National Natural Science Foundation of China (Grants 81030028, 30870667, 81020108022) and the Fundamental Research Funds for the Central Universities. A.C. was supported in part by the Fondazione Cassa di Risparmio di Trento e Rovereto.

The authors declare no competing financial interests.

Correspondence should be addressed to Yanchao Bi, State Key Laboratory of Cognitive Neuroscience and Learning, Beijing Normal University, Beijing 100875, China. E-mail: ybi@bnu.edu.cn.

DOI:10.1523/JNEUROSCI.1953-11.2012

Copyright $\odot 2012$ the authors $\quad 0270-6474 / 12 / 320481-09 \$ 15.00 / 0$ using resting-state functional magnetic resonance imaging (rsfMRI). We focus on object conceptual processing in relation to low-frequency $(<0.08 \mathrm{~Hz})$ fluctuations (LFFs) in the blood oxygen level-dependent (BOLD) signal at rest, given that these fluctuations are related to spontaneous neuronal activity (Logothetis et al., 2001; Raichle, 2006).

Previous investigations of the regional amplitude of the LFFs (ALFF) have found that the ALFF reflects physiological signals: the ALFF of gray matter is higher than that of the white matter (Biswal et al., 1995), the ALFF of the so-called "default mode network" (regions that are activate during resting state and deactivated during task performance) is higher than that of other regions (Zang et al., 2007), and individuals with cognitive brain disorders show abnormal ALFF in the regions critical for the corresponding cognitive processes (Zang et al., 2007; Hoptman et al., 2010). Healthy individuals' resting-state ALFFs correlate with task-evoked BOLD responses and with participants' behavioral measures (Mennes et al., 2011). Furthermore, high synchronization of LFFs between areas within the same neuroanatomical and/or functional systems has been reported (Biswal et al., 1995; Fox et al., 2005; Koyama et al., 2010). Significantly, the degree of such synchronization is associated with variability in healthy individuals' cognitive processing ability (e.g., reading ability) (Hampson et al., 2006), performance improvements (e.g., visualdetection performance) (Lewisa et al., 2009), and personality traits (e.g., autistic traits) (Di Martino et al., 2009). Thus, it ap- 
pears that regional activity amplitude and connectivity patterns of the LFFs are associated with aspects of cognitive functioning.

In this study, we examined the extent to which intrinsic brain activity (both regional activity and functional connectivity patterns) at rest predicts individual variation in semantic processing capacity: (1) we performed a correlation analysis between participants' performance on conceptual tasks and their regional ALFF to uncover potential core regions that could account for individual variation in semantic processing efficiency; (2) we defined such core regions as seeds and computed the functional connectivity strength between each seed and other voxels to reconstruct the brain network associated with the core regions, and then we assessed the predictive power of these connectivity strengths for subjects' semantic performance. We found that regional ALFF in the left (posterior) middle temporal gyrus (LMTG) and its functional connectivity with a set of other regions strongly predicted individual variation in conceptual processing capacity.

\section{Materials and Methods}

\section{Participants}

Thirty-four healthy, right-handed college students (20 females; $22.5 \pm$ 1.3 years old; range, 20 - 26 years old) with no history of neurological or psychiatric disorders were recruited from the campus of Beijing Normal University as paid participants, and written informed consents were obtained. This study was approved by the Institutional Review Board of the State Key Laboratory of Cognitive Neuroscience and Learning of Beijing Normal University.

\section{Image acquisition}

Images were acquired using a Siemens TRIO 3-Tesla scanner at the Beijing Normal University Imaging Center for Brain Research. The participants lay supine with their heads snugly fixed with straps and foam pads to minimize head movement. Functional images were obtained using an echoplanar imaging sequence with the following parameters: 33 axial slices; slice thickness, $3 \mathrm{~mm}$; gap, $0.6 \mathrm{~mm}$; repetition time (TR), $2000 \mathrm{~ms}$; echo time (TE), $30 \mathrm{~ms}$; voxel size, $3.1 \times 3.1 \times 3.5 \mathrm{~mm}$; flip angle, $90^{\circ}$; field of view (FOV), $200 \times 200 \mathrm{~mm}$; and 240 volumes. In addition, a T1-weighted sagittal three-dimensional magnetization-prepared rapid gradient echo sequence was acquired: 128 slices; TR, $2530 \mathrm{~ms}$; TE, $3.39 \mathrm{~ms}$; slice thickness, $1.33 \mathrm{~mm}$; voxel size, $1.3 \times 1.0 \times 1.3 \mathrm{~mm}$; flip angle, $7^{\circ}$; inversion time, $1100 \mathrm{~ms}$; FOV, $256 \times 256 \mathrm{~mm}$; and in-plane resolution, $256 \times 256$. During rs-fMRI scanning, participants were instructed to close their eyes, keep still, and not think about anything systematically or fall asleep.

\section{Behavioral tests}

To assess semantic processing ability, we focused on two commonly studied object categories: animals and artifacts. We selected three classical tasks that share semantic processing components but vary in the modalities of input and output: object picture naming, picture associative matching, and object sound naming. By considering these tasks both jointly and separately, we attempted to approximate the function of a shared semantic component and not just those of any specific input (e.g., visual) or output (e.g., oral naming) process. We further administered a series of other tasks to better establish that any potential results were indeed relevant to the semantic component in those tasks. Two baseline control tasks were used to regress out potential individual variation in peripheral motor and perceptual processing: a cued articulation task for the naming tasks and a shape-matching task for picture associative matching. We also performed a number judgment task to test whether the regions/networks were specific for semantic processing or general cognitive processing. The DMDX program (Forster and Forster, 2003) was used to present the stimuli and to record response latencies. The behavioral data were acquired about one year after image acquisition.

Object picture-naming task. Sixty color photographs of common objects ( 30 tools and 30 animals) were used. Participants were instructed to name the pictures as quickly as possible without making errors. Each trial began with the appearance of a fixation point ("+") on the center of the screen for $500 \mathrm{~ms}$, which was then replaced by a target picture. The picture disappeared when the participant produced a vocal response or when a $3 \mathrm{~s}$ deadline was reached. The next trial started $1 \mathrm{~s}$ later. Pictures from different categories were randomized, with no consecutive trials being semantically related.

Object picture associative-matching task. This task is a derivation of the Pyramid and Palm Trees Test (Howard and Patterson, 1992). Each trial consisted of a picture triplet with a reference picture (e.g., a hammer) displayed above a target (e.g., a nail) and a distracter picture (e.g., an axe). Participants needed to select the bottom picture that was more semantically related to the top picture. Seventy-two picture triplets were used, including 36 tool triplets and 36 animal triplets. The trial structure was as follows: A fixation point (“+”) was presented for $500 \mathrm{~ms}$, followed by a stimulus triplet. Participants responded by pressing a button box with two left-right aligned keys corresponding to the two alternative pictures. The triplets disappeared upon the response or after a $4 \mathrm{~s}$ deadline. The next trial started $1 \mathrm{~s}$ later.

Object sound-naming task. In this task, participants were required to name the object that produces the target sound (e.g., "dog" for a barking sound). Typical sounds of 20 tools and 20 animals were selected. Each trial started with a one s fixation point ("+"), followed by a sound stimulus. The mean duration of the sound stimuli was $3.38 \mathrm{~s}$ (range, $0.73-12.02 \mathrm{~s}$ ). The response deadline was $15 \mathrm{~s}$. The next trial was initiated by the experimenter manually upon hearing the participants' complete response.

Control tasks. Two nonsemantic control tasks that had similar task structures to the semantic tasks were used to regress out effects arising from general response latency differences. A cued articulation task consisting of 20 trials was the control task for the naming tasks (i.e., object picture naming and sound naming). Participants were asked to pronounce the sound "ah" as soon as they saw the fixation point ("+"). To discourage adoption of response strategies, we used three randomized trial intervals: 500, 1000, or $2000 \mathrm{~ms}$. A shape-matching task was the control task for the picture associative-matching task. The procedure was the same as for the picture associative-matching task, except that geometric shapes were presented, and the participants judged which of the two shapes in the bottom was identical to the top target shape by pressing the corresponding key. There were 36 trials.

Number judgment task. We administrated this task to assess the specificity of the results obtained for the object conceptual tasks. Each trial contained a reference number (e.g., 23) displayed above two other numbers (e.g., 21 and 29), and participants were required to choose which of the latter two numbers was closer in magnitude to the reference number. There were 50 trials, and all items were two-digit numbers.

\section{Data analysis procedures}

Behavioral data analyses. To correct for speed-accuracy trade-off effects, we used an inverse efficiency (IE) measure-the average response time of correct trials divided by accuracy - in the analyses of behavioral data (Townsend and Ashby, 1983). For normalization purposes, we computed the $z$ scores of each participant's IE; that is, we computed the mean IE for each participant and scaled each participant's mean IE using the SD of the mean IEs across participants. Then we reversed the sign of the IE $z$ scores so that higher scores would correspond to more efficient performance. This negated IE $z$ score was used as the index for behavioral capacity/efficiency throughout the analyses and is referred to as "efficiency score" below.

Functional imaging data preprocessing. Preprocessing was performed using Statistical Parametric Mapping software (SPM8; http://www.fil.ion.ucl.ac. $\mathrm{uk} / \mathrm{spm}$ ) and Data Processing Assistant for Resting-State fMRI (Yan and Zang, 2010). The first 10 volumes of the functional images were discarded for signal equilibrium and adaptation of the participants to the scanning noise. Next, slice timing and head motion correction were performed, and a mean functional image was obtained for each participant. No participant exhibited head motion of $>2 \mathrm{~mm}$ maximum translation or $2.5^{\circ}$ rotation throughout the course of scans. To normalize functional images, each participant's structural brain image was coregistered to the mean functional image and was subsequently segmented. The parameters obtained in segmentation were used to normalize each participant's functional image onto the Montreal Neurological Institute space (resampling voxel size was $3 \times 3 \times 3$ $\mathrm{mm}$ ). After the linear trend of the time courses was removed, a band- 
pass filter $(0.01-0.08 \mathrm{~Hz})$ was applied to reduce low-frequency drift and high-frequency noise. Finally, spatial smoothing ( $4 \mathrm{~mm}$ FWHM Gaussian kernel) was conducted to decrease spatial noise.

Because LFF is sensitive to signals in the gray matter, additional analyses were conducted on the gray matter mask generated using the following procedure. We included the voxels with a probability higher than 0.4 in the SPM5 template onto the gray matter mask. Given the signal distortion in cerebellum, we also excluded the cerebellar regions (\#91-\#116) in the Automated Anatomical Labeling template (Tzourio-Mazoyer et al., 2002). In total, there were 36,272 voxels in the gray matter mask.

Regional analysis: ALFF calculation and ALFF-behavior analysis procedures. Following Zang et al. (2007), the ALFF value of each voxel in the brain was extracted as the sum of amplitudes within the low-frequency range $(0.01-0.08 \mathrm{~Hz})$. Specifically, a filtered time series was transformed to the frequency domain to obtain a power spectrum for each voxel. Next, the square root was calculated at each frequency of the power spectrum. The averaged square root across $0.01-0.08 \mathrm{~Hz}$ at each voxel was taken as the ALFF. We scaled the ALFF values for each participant for standardization purposes: the mean ALFF value within the gray matter mask of the participant was computed, and then the ALFF of each voxel was divided by this mean ALFF value.

We used two methods to approximate the semantic behavioral performance from the three semantic tasks. We examined whether regional spontaneous activity was related to semantic processing capacity using measures derived from both methods. The first approach (Method 1) was to obtain a composite score using the three semantic tasks and to conduct correlation analyses between this composite semantic score and the ALFF (for a similar rationale, see Schwartz et al., 2009). The second approach (Method 2) was to consider the three semantic tasks separately and then to explore areas shared by these tasks.

In Method 1, we averaged the efficiency scores for the three semantic tasks to generate a semantic composite score for each participant, which was taken as an index of the participant's semantic processing capacity. We performed a regression analysis to reveal the relationship between ALFF and semantic performance while partialling out the contribution of general response latency differences. Specifically, we regressed out the efficiency scores on the cued articulation and shape-matching control tasks from the semantic composite efficiency score and then computed the correlation coefficient between the ALFF and the residualized semantic composite score. In Method 2, we explored any potential overlap among the areas associated with different semantic tasks to identify the common region(s) for all three semantic tasks. To obtain a correlation map for a specific semantic task, we calculated the correlations between the ALFF and the residualized efficiency score in this specific task after regressing out the efficiency scores in the corresponding control task. For the two naming tasks, the correlation map was obtained through a correlation between the ALFF and residualized efficiency scores in the naming task after regressing out the efficiency scores in the cued articulation task. Similarly, the correlation map for picture associative matching was obtained through a correlation between the ALFF and residualized efficiency scores in the picture associative-matching task after regressing out the efficiency scores in the shape-matching task. We then checked for the common regions for the three semantic tasks.

Network analysis: functional connectivity analysis and connectivity-behavior analysis procedures. On the basis of the results of regional ALFF analyses, we further explored whether any observed core region works in concert with other regions as a network for semantic processing. Our rationale was to first use the observed region(s) as seed(s) to perform functional connectivity analyses, mapping out the regions that function with the seeds as a network. Next, we examined whether or not any specific connections within this network were able to predict semantic behavior.

Before conducting functional connectivity analyses, six head motion parameters, white matter, and cerebrospinal signal were first regressed out. Functional connectivity analysis was performed using the RestingState fMRI Data Analysis Toolkit (REST; http://www.restfmri.net). A spherical seed ROI (radius, $6 \mathrm{~mm}$ ) was created, centering on the coordinates of each peak point identified in the regional ALFF-behavior correlation analysis. To obtain the functional connectivity map for each participant, we considered both of the two relevant measures: regression coefficient $(\beta)$ and correlation coefficient $(r)$. First, we calculated the mean time series of the seed ROI for each participant. For the same gray matter mask applied in the above regional analysis, we calculated the regression coefficients $(\beta)$ and correlation coefficients $(r)$ between the seed time series and other voxels to obtain a $\beta$ map and an $r$ map for each participant. Fisher $z$ score transformations were conducted for the correlation coefficients $(r)$ to generate a $z$-functional connectivity (FC) map for each participant. To identify the regions showing significant functional connectivity with the seed(s), we did one-sample $t$ tests on these individual $\beta$ maps or $z$-FC maps to see whether they were significantly different from zero ( $t>6.43$; corrected $p<0.01$; Bonferroni correction). For these regions, we performed correlation analyses between participants' semantic performance and $\beta$ values or Fisher $z$ scores while regressing out the peripheral response components reflected in the performance of the control tasks.

Validating the functional roles of the semantic network. To examine whether the regions found in the ALFF- and FC-behavior analyses are specific to (object) conceptual processing or are more generally involved in other cognitive processes, we conducted the following ROI analyses. First, these regions and/or networks were defined as ROIs, and the corresponding ALFF/FC values within each ROI were averaged. Then correlation analyses were performed between averaged ALFF/FC values and participants' behavioral indexes in the number judgment task.

Reconstructing the default mode network. We compared the obtained resting-state semantic network with the default mode network, which is active during the resting state, is deactivated by most cognitive tasks but not by semantic tasks (Raichle et al., 2001), and overlaps in part with semantic networks derived from task-based fMRI research (Binder et al., 2009). To identify the default mode network in our participant group, we performed functional connectivity analyses (both correlation coefficient and regression coefficient measures) based on one previously identified seed region located in posterior cingulate gyrus (PCG; radius, $6 \mathrm{~mm}$; central coordinates, -5 , $-49,40$ ) (Fox et al., 2005). Then, individuals' correlation coefficients were transformed into $z$-FC maps by the Fisher $z$ score transformation. Onesample $t$ tests were then performed on these individual $\beta$ maps and $z$-FC maps ( $t>6.43$; corrected $p<0.01$; Bonferroni correction).

Statistical analysis. AlphaSim was used to correct for multiple comparisons (originally in AFNI software and implemented in REST), and the corrected threshold was set at $p<0.05$. More specifically, the threshold of regional ALFF-behavior analysis was the combination of a voxelwise $p$ value of $<0.05$ and a cluster size of $>58$ voxels $(1566 \mathrm{~mm} 3)$. Because connectivity-behavior analyses were conducted on brain areas showing significant functional connectivity with the seeds, the threshold was a combination of a voxelwise $p$ value of $<0.05$ and a cluster size of $>45$ voxels (1215 mm3) (small volume correction).

\section{Results}

\section{Regional ALFF analysis and ALFF-behavior analysis}

Participants' mean response times, accuracies, and inverse efficiency values on the behavioral tasks are shown in Table 1.

To explore the association between semantic behavior and regional resting-state activity, we first correlated the averaged efficiency scores of the three semantic tasks (Method 1) with the ALFF value of each voxel across the whole brain. The ALFF of one region, the LMTG [peak, $-60,-48,-3 ; 3564 \mathrm{~mm}^{3}$ (132 voxels); Fig. $1 A]$, was positively correlated with participants' semantic performance $\left(r_{\text {peak }}=0.68 ; r_{\text {cluster }}=0.81 ; p\right.$ values $<0.001 ; r_{\text {peak }}$, correlation coefficient between the ALFF in the peak coordinate and behavioral scores; $r_{\text {cluster }}$, correlation coefficient between the averaged ALFF within the significant region and the behavioral scores; Fig. $1 B$ ). Thus, the regional ALFF variance of LMTG accounted for $66 \%\left(R^{2}\right)$ of the variation in semantic task performance across individuals.

When we conducted separate analyses on the three semantic tasks (Method 2), the LMTG was the only brain region in which the ALFF was significantly positively correlated with (1) picturenaming performance with cued articulation performance regressed out [peak, $-60,-48,-3 ; 2268 \mathrm{~mm}^{3}$ (84 voxels); $r_{\text {peak }}=$ 
$0.71 ; r_{\text {cluster }}=0.73 ; p$ values $\left.<0.001\right]$ and (2) picture associativematching performance with shape-matching performance regressed out [peak, $-66,-45,-3 ; 3375 \mathrm{~mm}^{3}$ (125 voxels); $r_{\text {peak }}=$ $0.81 ; r_{\text {cluster }}=0.59 ; p$ values $\left.<0.001\right]$. In the object soundnaming task, we found positive correlations in right superior temporal and inferior parietal lobes [peak, 54, - 36, 24; 2511 $\mathrm{mm}^{3}$ (93 voxels); $r_{\text {peak }}=0.67 ; r_{\text {cluster }}=0.67 ; p$ values $\left.<0.001\right]$. It should be noted that the particularly low levels of accuracy and large variance in performance in this task may have originated in part at the object sound perceptual/recognition phase. Since we did not include a control task for this phase of the object soundnaming task, we lowered the threshold to corrected $p<0.1$ [a combination of a voxelwise $p$ value of $<0.05$ and a cluster size of $>1377 \mathrm{~mm}^{3}$ (51 voxels)]. We found that, with cued articulation regressed out, LMTG showed association with object sound-naming performance [peak, -66 , $-42,-6 ; 1431 \mathrm{~mm}^{3}$ (53 voxels); $r_{\text {peak }}=$ $0.59 ; r_{\text {cluster }}=0.64 ; p$ values $\left.<0.001\right]$.

Together, the findings obtained with the two methods converge in showing that the LMTG is relevant for semantic processing regardless of stimulus input and output modality.

\section{Functional connectivity analysis and connectivity-behavior analysis}

To explore whether LMTG functions in concert with other brain regions for semantic processing, we performed a seed voxel correlation analysis between the LMTG region found with the semantic composite score (Method 1) and all other voxels in the brain. As seen in Figure 2, $A$ and $B$, we found similar functional connectivity patterns using the regression coefficient and correlation coefficient measures. A wide range of brain regions showed significant functional connectivity with LMTG, including the bilateral temporal gyri, inferior occipital gyri, middle and inferior frontal gyri, precentral cortices, ventromedial and dorsomedial frontal cortices, angular gyri, and posterior cingulate gyri. We defined these regions as a large ROI mask and correlated each voxel value within this ROI with semantic composite scores across participants. For the regression coefficient approach, the strength of functional connectivity between LMTG and the following regions significantly predicted participants' semantic performance: left inferior frontal gyrus (LIFG), bilateral lateral temporal lobe including anterior temporal lobe (ATL), left medial temporal lobe (LMTL), PCG, dorsomedial prefrontal cortex (DMPFC), and ventromedial prefrontal cortex (VMPFC) (Fig. 3; Table 2). When the correlation coefficients were considered, significant correlations were also observed between functional connectivity in most of these regions and LMTG and participants' semantic performance (Table 2).

We performed ROI analyses to further examine whether the regional activity of these regions (in the regression coefficient results) correlated with semantic processing performance. First, the averaged ALFF value within each region was obtained. Then correlation analyses between participants' semantic composite scores and averaged ALFF values for each region were performed. No region showed significant effects in these ROI analyses ( $r$ values $<0.27$; $p$ values $>0.12$ ).
Table 1. Participants' performance on behavioral tasks

\begin{tabular}{|c|c|c|c|c|c|c|}
\hline \multirow[b]{2}{*}{ Tasks } & \multicolumn{2}{|c|}{$\begin{array}{l}\text { Response time } \\
\text { (ms) }\end{array}$} & \multicolumn{2}{|c|}{ Accuracy } & \multicolumn{2}{|c|}{$\begin{array}{l}\text { Inverse } \\
\text { efficiency }\end{array}$} \\
\hline & Mean & SD & Mean & SD & Mean & SD \\
\hline \multicolumn{7}{|l|}{ Semantic tasks } \\
\hline $\begin{array}{l}\text { Object picture-naming } \\
\text { Object picture associative- }\end{array}$ & 1082 & 152 & $94 \%$ & $4 \%$ & 1149 & 187 \\
\hline matching & 1608 & 243 & $91 \%$ & $6 \%$ & 1781 & 292 \\
\hline Object sound-naming & 2857 & 506 & $76 \%$ & $10 \%$ & 3832 & 968 \\
\hline \multicolumn{7}{|l|}{ Other tasks } \\
\hline Cued articulation & 376 & 91 & $100 \%$ & $0 \%$ & 376 & 91 \\
\hline Shape matching & 487 & 72 & $96 \%$ & $4 \%$ & 506 & 64 \\
\hline Number judgment & 1316 & 310 & $93 \%$ & $4 \%$ & 1409 & 317 \\
\hline
\end{tabular}

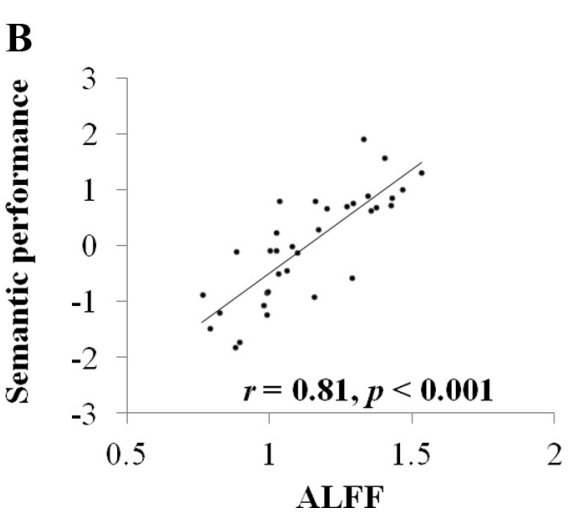

Figure 1. Regional ALFF-behavior correlation analyses using composite semantic scores. $\boldsymbol{A}$, Statistical map for the correlation 列 cued articulation and shape-matching control tasks were regressed out from the semantic composite efficiency score. Each dot represents data from one participant.

Combining regional- and connectivity-behavior analyses

To assess the joint contributions of the regional activity and connectivity of LMTG in predicting semantic behavior, we performed a multiple linear regression analysis. The dependent variable was participants' semantic composite scores after regressing out the efficiency scores on the cued articulation and shape-matching control tasks. Independent variables included the ALFF values of LMTG and seven functional connectivity variables. The ALFF variable was obtained by averaging ALFF values within the LMTG region that was significant in the regional analysis (Method 1). The functional connectivity variables were extracted by averaging the regression coefficients within each observed region in the functional connectivity-behavior analyses. When all independent variables were simultaneously entered into the regression, the regression model explained $74 \%$ of the variation in participants' semantic processing performance $\left(R^{2}=\right.$ $\left.0.74 ; F_{(8,25)}=8.96 ; p<0.001\right)$. We also used a forward method to test how much independent contribution each of the two types of variables provided. When the ALFF variable was entered first and the 10 functional connectivity variables were entered in the second step, we found that the inclusion of the functional connectivity variables yielded a trend of improvement for the explanatory power of the regression model $\left(R^{2}=\right.$ $0.66 \rightarrow 0.74)$, but the effect was not significant $\left(F_{(7,25)}=1.18\right.$; $p=0.35)$. When the functional connectivity variables were entered first, the addition of ALFF into the model yielded significant $R^{2}$ change $\left(R^{2}=0.49 \rightarrow 0.74 ; F_{(1,25)}=24.18 ; p<\right.$ $0.001)$. These results further highlight the central role of LMTG's regional activity in predicting semantic performance. 
A

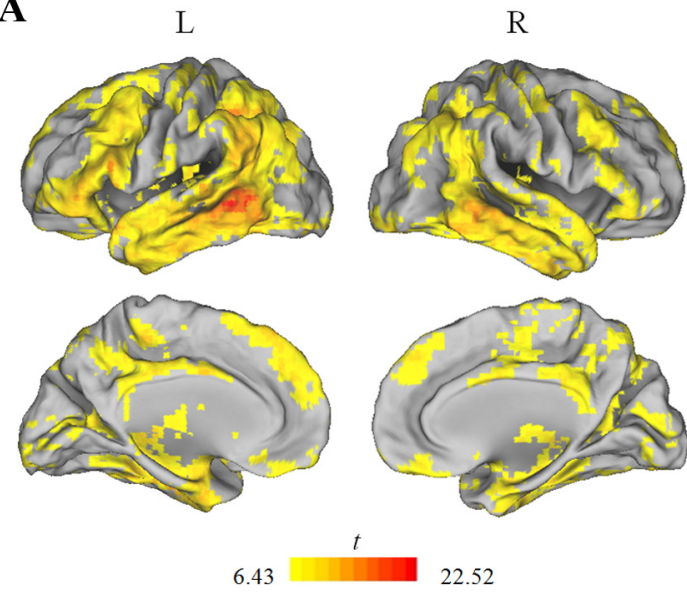

B
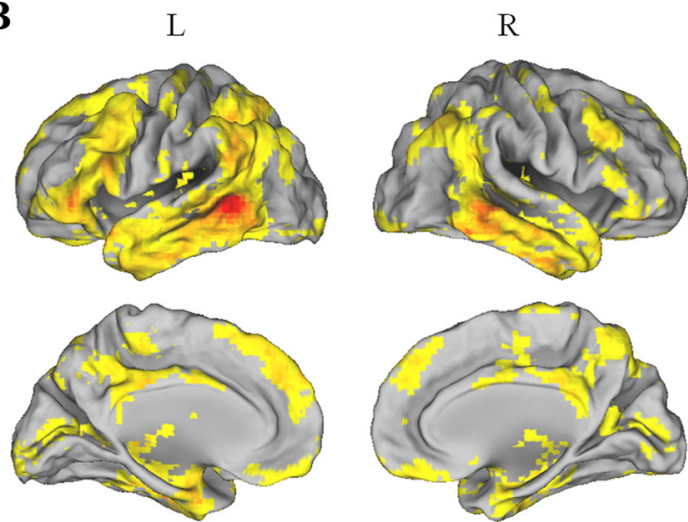

6.43

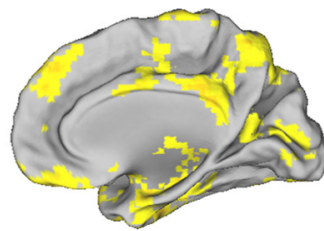

27.68

Figure 2. $\quad \boldsymbol{A}, \boldsymbol{B}$, Statistical maps of functional connectivity of LMTG with the regression coefficient $(\boldsymbol{A})$ and correlation coefficient $(\boldsymbol{B})$ measures: voxels for which the time series showed a significant association with the seed ROl in LMTG.

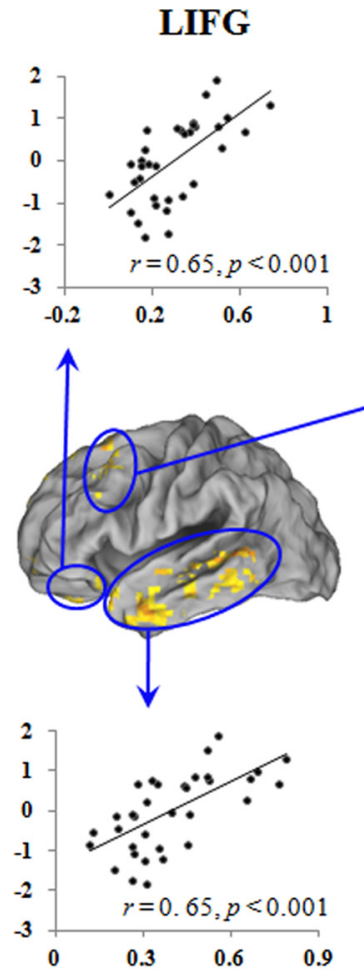

Left MTG
DMPFC
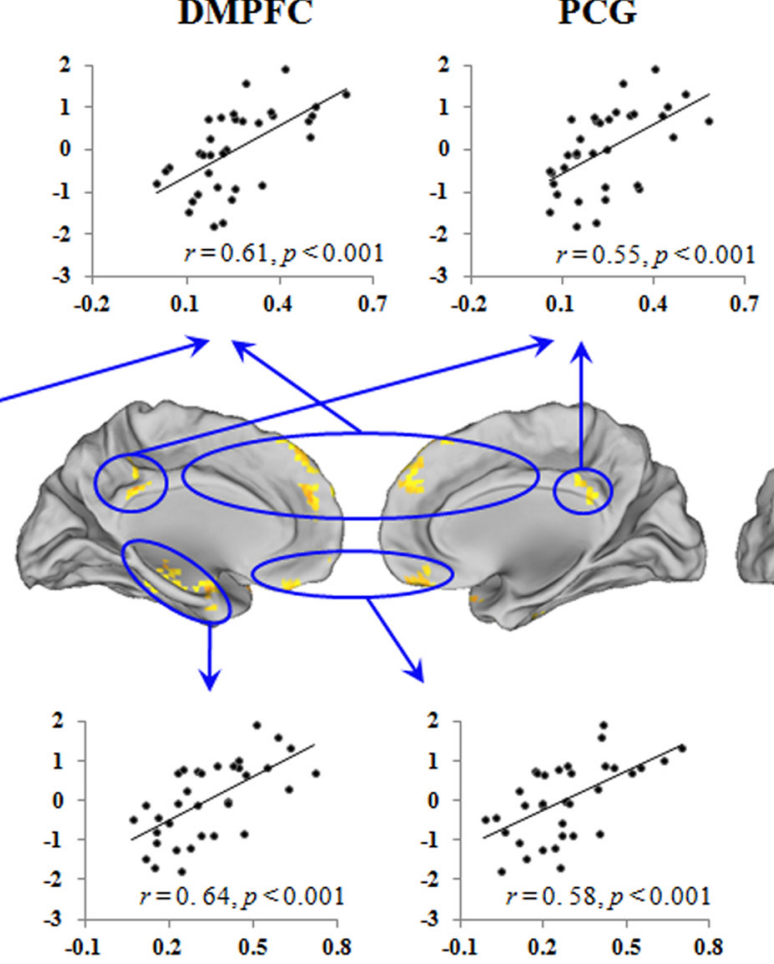

Left MTL
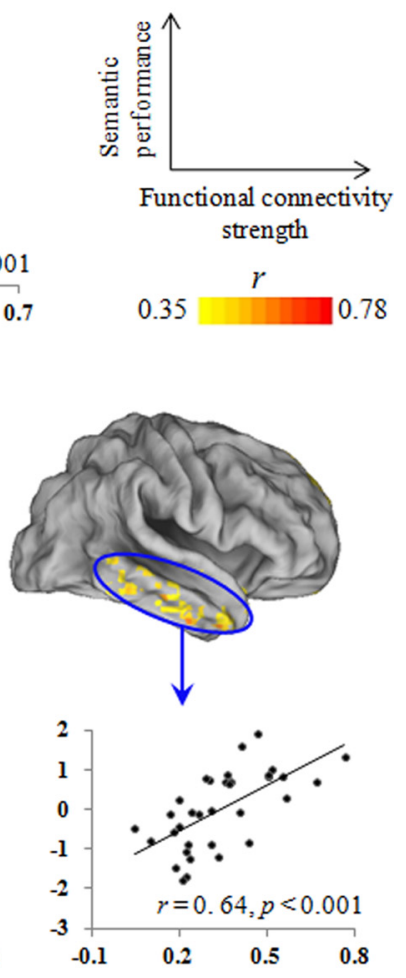

Right MTG

Figure 3. Clusters for which functional connectivity strength $(\beta)$ with LMTG significantly predicted semantic composite scores. Scatter plots show positive correlations between participants' semantic performance and functional connectivity strength between LMTG and the clusters in the blue circles, while the efficiency scores on the cued articulation and shape-matching control tasks were regressed out from the semantic composite efficiency score. Each dot represents data from one participant.

The functional specificity of the observed semantic network The results of ROI analyses for the number judgment task are shown in Table 3. ${ }^{a}$ Within the LMTG network we obtained for the semantic tasks, we observed that the intrinsic brain activity (i.e., both regional activity and functional connectivity) was not significantly associated with the number-processing task. These

${ }^{a}$ We also performed whole-brain correlation analyses between the regional activity and efficiency scores in the number judgment task. We observed a significant association between participants' behavior and intrinsic activity in left inferior and superior parietal lobule encompassing the left intraparietal sulcus [peak coordinates (range), $-51(-52 \sim-17),-48(-59 \sim-38), 54(47 \sim 67) ; 57$ voxels; corrected $p<0.05$ ], close to the number regions reported in previous studies (Dehaene et al., 2003). results indicate that different domains of conceptual processing (object vs numbers) can be distinguished.

\section{Default mode network and semantic network}

As shown in Figure $4 A$, bilateral inferior parietal lobe, medial prefrontal cortex, superior frontal cortex, medial temporal lobe, and lateral temporal cortex had strong functional connectivity with PCG, and these regions constitute the default mode network. Figure $4 B$ replots the data presented in Figures 3 and $4 A$, showing that the semantic network largely overlaps with the default mode network. 
Table 2. Clusters for which functional connectivity strength with LMTG can predict semantic performance

\begin{tabular}{|c|c|c|c|c|c|c|c|c|c|c|c|c|}
\hline \multirow[b]{3}{*}{ Brain regions } & \multicolumn{6}{|c|}{ Regression coefficient } & \multicolumn{6}{|c|}{ Correlation coefficient } \\
\hline & \multirow[b]{2}{*}{ BA } & \multicolumn{3}{|c|}{ Peak MNI coordinates } & \multirow[b]{2}{*}{$r(p)$ (peak) } & \multirow[b]{2}{*}{ Volumes $\left(\mathrm{mm}^{3}\right)$} & \multirow[b]{2}{*}{$\mathrm{BA}$} & \multicolumn{3}{|c|}{ Peak MNI coordinates } & \multirow[b]{2}{*}{$r(p)$ (peak) } & \multirow[b]{2}{*}{ Volumes $\left(\mathrm{mm}^{3}\right)$} \\
\hline & & $x$ & $y$ & $z$ & & & & $x$ & $y$ & $z$ & & \\
\hline LIFG & 11,47 & -30 & 54 & -12 & $0.78(<0.001)$ & 2943 & 11,47 & -30 & 54 & -12 & $0.62(<0.001)$ & 1593 \\
\hline Left MTG & $38,37,21,20$ & -54 & -54 & 3 & $0.71(<0.001)$ & 16038 & 20,21 & -54 & -39 & -6 & $0.46(<0.01)$ & 1593 \\
\hline Right MTG & $38,37,21,20$ & 51 & 18 & -21 & $0.67(<0.001)$ & 11475 & 38,21 & 45 & 3 & -33 & $0.57(<0.001)$ & 2646 \\
\hline Left MTL & 20,37 & -30 & -9 & -18 & $0.64(<0.001)$ & 2565 & 20 & -28 & -20 & -11 & $0.44(<0.01)$ & $324^{a}$ \\
\hline$P C G$ & 23 & -3 & -42 & 30 & $0.59(<0.001)$ & 1593 & 23 & 1 & -41 & 30 & $0.40(<0.05)$ & $270^{a}$ \\
\hline DMPFC & $8,9,10$ & 15 & 63 & 21 & $0.65(<0.001)$ & 9936 & 9 & 6 & 51 & 45 & $0.53(<0.01)$ & 1647 \\
\hline VMPFC & 11 & 3 & 48 & -15 & $0.58(<0.001)$ & 1755 & 11 & 18 & 60 & -15 & $0.50(<0.01)$ & 1296 \\
\hline
\end{tabular}

For the regression coefficients $(\beta)$ results, a $\beta$ map was generated for each participant by calculating the regression efficient between the seed time series and other voxels; then one sample $t$ test on these individual $\beta$ maps to see whether they were significantly different from zero ( $t>6.43$, corrected $p<0.01$, Bonferroni correction); then for the significant regions, correlation analyses between participants' semantic performance and $\beta$ values were conducted while regressing out the peripheral response components reflected in the performance of the control tasks. The correlation coefficient $(r)$ results were obtained using the same procedure except that correlation efficient between the seed time series and other voxels were considered and that Fisher $z$-score transformation was performed on these $r$ values.

${ }^{a}$ The peak voxel of these clusters reached the threshold of $p<0.05$, but they did not survive multiple comparison correction [cluster size $>38$ voxels $\left(1026 \mathrm{~mm}^{3}\right)$ ].

Table 3. The results of ROI analysis for the number judgment task

\begin{tabular}{lr}
\hline ROl regions & Correlation $r(p)$ \\
\hline Regional activity & \\
LMTG & $0.04(0.82)$ \\
functional connectivity & \\
LIFG & $0.03(0.87)$ \\
LMTG (including ATL) & $-0.03(0.37)$ \\
Right MTG (including ATL) & $-0.07(0.69)$ \\
LMTL & $-0.18(0.30)$ \\
PCG & $-0.08(0.65)$ \\
DMPFC & $0.02(0.91)$ \\
VMPFC & $-0.11(0.54)$ \\
\hline
\end{tabular}

\section{Discussion}

We observed that the amplitude of resting-state LFF activity in LMTG accounted for $66 \%$ of individual variance in object conceptual processing efficiency. This region functions together with six other regions in a semantic memory network: we found that the strength of functional connectivity between LMTG and these regions (LIFG, bilateral ATL, PCG, bilateral MTL, VMPFC, and DMPFC) also significantly predicted participants' semantic processing efficiency. Regression analysis revealed that this network, with LMTG as its core component, accounted for $74 \%$ of semantic processing performance variation, but did not correlate with participants' performance on a non-object-conceptual task (i.e., number processing).

The LMTG network identified here largely overlaps with regions that have been previously implicated in semantic processing tasks in the functional brain-imaging literature. LMTG has long been observed to be important for semantic processing, and all seven regions obtained in the network analysis overlap with the regions that were reported in a previous meta-analysis of task-based fMRI and positron emission tomography studies of semantic processing (Binder et al., 2009). The only difference is that the meta-analysis reported a significant role for the angular gyrus, while this area was not found in our analysis.

\section{LMTG as a core semantic region}

The LMTG region identified here corresponds to the middle part of Brodmann's area (BA) 21, extending to BA 37. In brainimaging studies, the LMTG has been found to be activated by tasks that probe semantic processing through various modalities, including visual words and pictures (Vandenberghe et al., 1996), auditory words (Hickok and Poeppel, 2004), and tactile inputs (Stoeckel et al., 2003). Task-induced activity in the LMTG has also been observed for semantic information acquisition relative to baseline (Maguire and Frith, 2004). The essential role of the LMTG in word-level comprehension is also indicated by lesionsymptom mapping analysis on stroke patients (Bates et al., 2003; Dronkers et al., 2004; Schwartz et al., 2009) and voxel-based morphometric analyses on semantic dementia patients (Mummery et al., 2000). Turken and Dronkers (2011) showed previously that the LMTG has rich structural and spontaneous functional connectivity with other regions relevant for language comprehension, and they proposed that it plays a central role in comprehension.

In some task-based fMRI and lesion studies (Damasio et al., 1996; Martin et al., 1996), the LMTG has been shown to be more sensitive to certain semantic categories such as tools, while other studies have observed effects for a wide range of semantic categories (Rudrauf et al., 2008; Simmons et al., 2010), including verbs (Willms et al., 2011), suggesting that the effect reported here is not likely to be driven only by tool items. We nevertheless analyzed tool and animal items separately in our study, and found similar LMTG effects for the two categories [for tools, peak, -63 , $-45,-3 ; 3024 \mathrm{~mm}^{3}$ (112 voxels); $r_{\text {peak }}=0.64 ; r_{\text {cluster }}=0.77 ; p$ values $<0.001$; for animals, peak, $-60,-48,-3 ; 3186 \mathrm{~mm}^{3}(118$ voxels $) ; r_{\text {peak }}=0.63 ; r_{\text {cluster }}=0.74 ; p$ values $\left.<0.001\right]$. It is possible that the tool-specific effects reported in the literature originated from an LMTG region that was close to but different from ours (Simmons et al., 2010). We did show, however, that number judgment performance did not correlate with the observed object semantic LMTG network, but with parietal regions close to the classical number regions (Footnote $a$ ), suggesting that our technique has the potential to distinguish between conceptual domains.

Together, these findings constitute clear evidence for a critical role of the LMTG in semantic processing, at least for single object concepts (Lau et al., 2008). The present study further strengthens this view by showing the robust effect of the spontaneous activity of this region in predicting individual participants' variation in semantic processing behavior.

\section{Regions for which connectivity with LMTG predicted semantic processing behavior}

Among the brain regions showing resting-state synchronization with the LMTG, a subset of these regions further predicted variation in semantic processing behavior, although their contribution beyond the regional ALFF effect of the LMTG was not significant. Among these regions, the LIFG and left ATL have consistently been observed to have white matter fiber pathways 
A
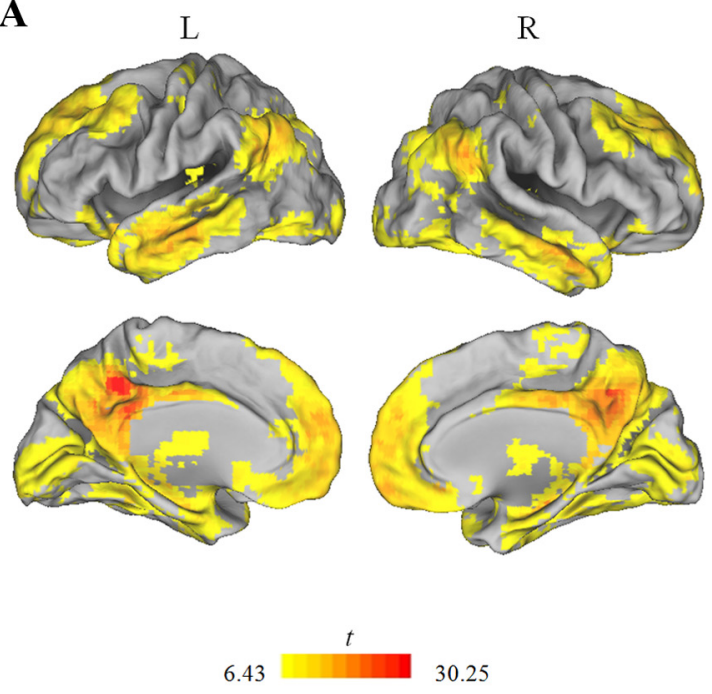

B
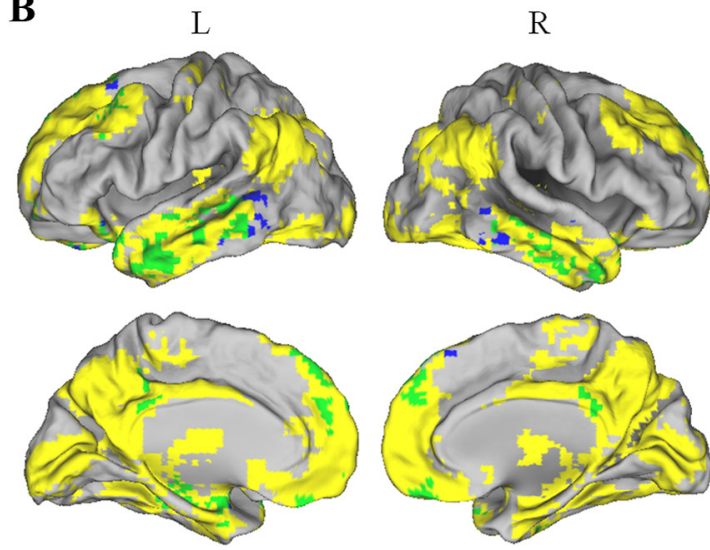

Default mode network

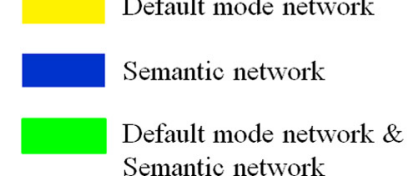

Figure 4. Default mode network and its comparison with the observed semantic network. $A$, Default mode network results. The bilateral inferior parietal lobe, medial prefrontal cortex, superior frontal cortex, medial temporal lobe, and lateral temporal cortex had strong functional connectivity with PCG, and these regions constitute the default mode network. $\boldsymbol{B}$, Overlay of Figure 3 and $\boldsymbol{A}$, showing that the semantic network lies within the default mode network.

(i.e., anatomical connectivity) projecting to the LMTG in diffusion tensor imaging (DTI) and anatomical dissection studies (Catani et al., 2005; Catani and Mesulam, 2008; Papagno et al., 2011; Turken and Dronkers, 2011). Importantly, these fiber bundles seem to be specifically engaged in semantic and not phonological tasks (Saur et al., 2008, 2010). Furthermore, LIFG, especially the specific region implicated in the current study (BA 47 ), is activated by a variety of semantic tasks (Fiez, 1997; Poldrack et al., 1999; Friederici et al., 2003). The involvement of the ATL in semantic processing is most strongly suggested by semantic dementia patients, who suffer from atrophy of the ATL accompanied by progressive semantic impairment (for review, see Patterson et al., 2007).

Using DTI, Gong et al. (2009) reported that white matter fiber bundles exist between the LMTG and PCG. PCG has been shown to be associated with various cognitive functions, including episodic retrieval (Wagner et al., 2005), self-monitoring (Vogt and Laureys, 2005), self-reflection (Johnson et al., 2002), and awareness (Vogt and Laureys, 2005). We speculate that semantic processing might induce episodic encoding and thus can lead to PCG engagement.

The anatomical connections between the other three regions (MTL, VMPFC, and DMPFC) and LMTG are far from clear. Neurophysiological, neuroimaging, and neuropsychological studies provide converging evidence that the MTL is critical for semantic processing, especially the acquisition and retrieval of semantic memory (for review, see Squire et al., 2004). VMPFC has been strongly linked to social cognition. Specifically, it has been ascribed a crucial role for the encoding of enduring social dispositions and interpersonal knowledge through the integration of social information over long stretches of time in multiple circumstances (Van Overwalle, 2009). Such processes may be shared by semantic memory, which also implicates the abstraction of consistent information from disparate instances of an object or event and the decontextualization of individual memories (Takashima et al., 2006). The role of DMPFC is less clear, but, as speculated by Binder et al. (2009), it might be involved in "self-guided retrieval" aspects of semantic processing. Lesions to this region have been found to induce symptoms of very little spontaneous speech or action despite the preserved ability to speak or act when prompted (Nagaratnam et al., 2004).

Finally, the resting-state semantic network observed here largely overlaps with the default mode network (Shulman et al., 1997; Raichle et al., 2001). It has been proposed that during its conscious resting state, this network reflects ongoing semantic processing, such as semantic knowledge retrieval and manipulation of represented knowledge for problem solving (Binder et al., 2009). Other researchers have linked the network's activity to internally generated thought processes related to self-reflection (Gusnard et al., 2001) or mind wandering (Mason et al., 2007) processes that implicate a critical role for semantics. Our results further reinforce the notion that the function of the default mode network involves semantic processing.

\section{Potential mechanisms for the rs-fMRI-behavior correlation}

The neuronal origin of rs-fMRI activity has been supported by many types of findings, including its correlation with electrophysiological recordings of neuronal firing (Nir et al., 2008), structural connectivity, slow cortical potentials, and the bandlimited power of fast electrical activity (for review, see Zhang and Raichle, 2010). The correlation between rs-fMRI and behavioral efficiency reported here might be driven by either genetic and/or environmental variables. The influence of genetic factors on specific rs-fMRI patterns has been demonstrated (Glahn et al., 2010), and it is not implausible to assume that variation in genetic disposition may optimize brain regions/networks to acquire and process semantic knowledge most efficiently. On the other hand, training has also been shown to affect rs-fMRI patterns (Lewisa et al., 2009), and it is possible that the richness of semantic experience shapes the rs-fMRI patterns in relevant regions. Future studies should consider the relative contribution of these variables in determining rs-fMRI patterns. 
In conclusion, we observed a network, with the LMTG as its core component, in which the resting-state activity predicts healthy individuals' conceptual processing efficiency. This finding has multifaceted theoretical implications: (1) it further confirms the roles of these regions, especially the central role of LMTG, in object conceptual processing; (2) it reveals a potential new mechanism for the representation and "maintenance" of semantic memory (i.e., through resting-state fluctuations); and (3) the extensive overlap between the resting state of the semantic network and the default mode network reinforces previous claims that the function of the default mode network may involve semantic processing. Finally, our findings have important clinical implications, providing potential biomarkers for the early detection of conceptual knowledge deterioration.

\section{References}

Bates E, Wilson SM, Saygin AP, Dick F, Sereno MI, Knight RT, Dronkers NF (2003) Voxel-based lesion-symptom mapping. Nat Neurosci 6:448-450.

Binder JR, Desai RH, Graves WW, Conant LL (2009) Where is the semantic system? A critical review and meta-analysis of 120 functional neuroimaging studies. Cereb Cortex 19:2767-2796.

Biswal B, Yetkin FZ, Haughton VM, Hyde JS (1995) Functional connectivity in the motor cortex of resting human brain using echo-planar MRI. Magn Reson Med 34:537-541.

Catani M, Mesulam M (2008) The arcuate fasciculus and the disconnection theme in language and aphasia: history and current state. Cortex 44:953-961.

Catani M, Jones DK, Ffytche DH (2005) Perisylvian language networks of the human brain. Ann Neurol 57:8-16.

Damasio H, Grabowski TJ, Tranel D, Hichwa RD, Damasio AR (1996) A neural basis for lexical retrieval. Nature 380:499-505.

Dehaene S, Piazza M, Pinel P, Cohen L (2003) Three parietal circuits for number processing. Cogn Neuropsychol 20:487-506.

Di Martino A, Shehzad Z, Kelly C, Roy AK, Gee DG, Uddin LQ, Gotimer K, Klein DF, Castellanos FX, Milham MP (2009) Relationship between cingulo-insular functional connectivity and autistic traits in neurotypical adults. Am J Psychiatry 166:891-899.

Dronkers NF, Wilkins DP, Van Valin RD Jr, Redfern BB, Jaeger JJ (2004) Lesion analysis of the brain areas involved in language comprehension. Cognition 92:145-177.

Fiez JA (1997) Phonology, semantics, and the role of the left inferior prefrontal cortex. Hum Brain Mapp 5:79-83.

Forster KI, Forster JC (2003) DMDX: a windows display program with millisecond accuracy. Behav Res Methods Instrum Comput 35:116-124.

Fox MD, Snyder AZ, Vincent JL, Corbetta M, Van Essen DC, Raichle ME (2005) The human brain is intrinsically organized into dynamic, anticorrelated functional networks. Proc Natl Acad Sci U S A 102:9673-9678.

Friederici AD, Ruschemeyer SA, Hahne A, Fiebach CJ (2003) The role of left inferior frontal and superior temporal cortex in sentence comprehension: localizing syntactic and semantic processes. Cereb Cortex 13:170-177.

Glahn DC, Winkler AM, Kochunov P, Almasy L, Duggirala R, Carless MA, Curran JC, Olvera RL, Laird AR, Smith SM, Beckmann CF, Fox PT, Blangero J (2010) Genetic control over the resting brain. Proc Natl Acad Sci U S A 107:1223-1228.

Gong GL, He Y, Concha L, Lebel C, Gross DW, Evans AC, Beaulieu C (2009) Mapping anatomical connectivity patterns of human cerebral cortex using in vivo diffusion tensor imaging tractography. Cereb Cortex 19:524-536.

Gusnard D, Akbudak E, Shulman G, Raichle ME (2001) Role of medial prefrontal cortex in a default mode of brain function. Neuroimage 13:S414-S414.

Hampson M, Tokoglu F, Sun ZD, Schafer RJ, Skudlarski P, Gore JC, Constable RT (2006) Connectivity-behavior analysis reveals that functional connectivity between left BA39 and Broca's area varies with reading ability. Neuroimage 31:513-519.

Hickok G, Poeppel D (2004) Dorsal and ventral streams: a framework for understanding aspects of the functional anatomy of language. Cognition 92:67-99.

Hoptman MJ, Zuo XN, Butler PD, Javitt DC, D’Angelo D, Mauro CJ, Milham
MP (2010) Amplitude of low-frequency oscillations in schizophrenia: a resting state fMRI study. Schizophr Res 117:13-20.

Howard D, Patterson KE (1992) The pyramids and palm trees test: a test of semantic access from words and pictures. Bury St. Edmunds, UK: Thames Valley Test Company.

Johnson SC, Baxter LC, Wilder LS, Pipe JG, Heiserman JE, Prigatano GP (2002) Neural correlates of self-reflection. Brain 125:1808-1814.

Koyama MS, Kelly C, Shehzad Z, Penesetti D, Castellanos FX, Milham MP (2010) Reading networks at rest. Cereb Cortex 20:2549-2559.

Lau EF, Phillips C, Poeppel D (2008) A cortical network for semantics: (de)constructing the N400. Nat Rev Neurosci 9:920-933.

Lewisa CM, Baldassarre A, Committeri G, Romani GL, Corbetta M (2009) Learning sculpts the spontaneous activity of the resting human brain. Proc Natl Acad Sci U S A 106:17558-17563.

Logothetis NK, Pauls J, Augath M, Trinath T, Oeltermann A (2001) Neurophysiological investigation of the basis of the fMRI signal. Nature 412:150-157.

Maguire EA, Frith CD (2004) The brain network associated with acquiring semantic knowledge. Neuroimage 22:171-178.

Martin A, Wiggs CL, Ungerleider LG, Haxby JV (1996) Neural correlates of category-specific knowledge. Nature 379:649-652.

Mason MF, Norton MI, Van Horn JD, Wegner DM, Grafton ST, Macrae CN (2007) Wandering minds: the default network and stimulus-independent thought. Science 315:393-395.

Mennes M, Zuo XN, Kelly C, Di Martino A, Zang YF, Biswal B, Castellanos FX, Milham MP (2011) Linking inter-individual differences in neural activation and behavior to intrinsic brain dynamics. Neuroimage 54:2950-2959.

Mummery CJ, Patterson K, Price CJ, Ashburner J, Frackowiak RSJ, Hodges JR (2000) A voxel-based morphometry study of semantic dementia: relationship between temporal lobe atrophy and semantic memory. Ann Neurol 47:36-45

Nagaratnam N, Nagaratnam K, Ng K, Diu P (2004) Akinetic mutism following stroke. J Clin Neurosci 11:25-30.

Nir Y, Mukamel R, et al (2008) Interhemispheric correlations of slow spontaneous neuronal fluctuations revealed in human sensory cortex. Nat Neurosci 11:1100-1108.

Papagno C, Gallucci M, Casarotti A, Castellano A, Falini A, Fava E, Giussani C, Carrabba G, Bello L, Caramazza A (2011) Connectivity constraints on cortical reorganizationof neural circuits involved in object naming. Neuroimage 55:1306-1313.

Patterson K, Nestor PJ, Rogers TT (2007) Where do you know what you know? The representation of semantic knowledge in the human brain. Nat Rev Neurosci 8:976-987.

Poldrack RA, Wagner AD, Prull MW, Desmond JE, Glover GH, Gabrieli JDE (1999) Functional specialization for semantic and phonological processing in the left inferior prefrontal cortex. Neuroimage 10:15-35.

Raichle ME (2006) The brain's dark energy. Science 314:1249-1250.

Raichle ME, MacLeod AM, Snyder AZ, Powers WJ, Gusnard DA, Shulman GL (2001) A default mode of brain function. Proc Natl Acad Sci U S A 98:676-682.

Rudrauf D, Mehta S, Bruss J, Tranel D, Damasio H, Grabowski TJ (2008) Thresholding lesion overlap difference maps: application to categoryrelated naming and recognition deficits. Neuroimage 41:970-984.

Saur D, Kreher BW, Schnell S, Kummerer D, Kellmeyer P, Vry MS, Umarova R, Musso M, Glauche V, Abel S, Huber W, Rijntjes M, Hennig J, Weiller C (2008) Ventral and dorsal pathways for language. Proc Natl Acad Sci U S A 105:18035-18040.

Saur D, Schelter B, Schnell S, Kratochvil D, Kupper H, Kellmeyer P, Kummerer D, Kloppel S, Glauche V, Lange R, Mader W, Feess D, Timmer J, Weiller C (2010) Combining functional and anatomical connectivity reveals brain networks for auditory language comprehension. Neuroimage 49:3187-3197.

Schwartz MF, Kimberg DY, Walker GM, Faseyitan O, Brecher A, Dell GS, Coslett HB (2009) Anterior temporal involvement in semantic word retrieval: voxel-based lesion-symptom mapping evidence from aphasia. Brain 132:3411-3427.

Shulman GL, Fiez JA, Corbetta M, Buckner RL, Miezin FM, Raichle ME, Petersen SE (1997) Common blood flow changes across visual tasks. 2. Decreases in cerebral cortex. J Cognitive Neurosci 9:648-663. 
Simmons WK, Reddish M, Bellgowan PSF, Martin A (2010) The selectivity and functional connectivity of the anterior temporal lobes. Cereb Cortex 20:813-825

Squire LR, Stark CE, Clark RE (2004) The medial temporal lobe. Annu Rev Neurosci 27:279-306.

Stoeckel MC, Weder B, Binkofski F, Buccino G, Shah NJ, Seitz RJ (2003) A fronto-parietal circuit for tactile object discrimination: an event-related fMRI study. Neuroimage 19:1103-1114.

Takashima A, Petersson KM, Rutters F, Tendolkar I, Jensen O, Zwarts MJ, McNaughton BL, Fernandez G (2006) Declarative memory consolidation in humans: a prospective functional magnetic resonance imaging study. Proc Natl Acad Sci U S A 103:756-761.

Townsend JT, Ashby FG (1983) Stochastic modelling of elementary psychological processes. New York: Cambridge UP.

Tulving E (1972) Organization of memory (Tulving E, Donaldson W, eds). London: Academic.

Turken AU, Dronkers NF (2011) The neural architecture of the language comprehension network: converging evidence from lesion and connectivity analyses. Front Syst Neurosci 5:1.

Tzourio-Mazoyer N, Landeau B, Papathanassiou D, Crivello F, Etard O, Delcroix N, Mazoyer B, Joliot M (2002) Automated anatomical labeling of activations in SPM using a macroscopic anatomical parcellation of the MNI MRI single-subject brain. Neuroimage 15:273-289.

Vandenberghe R, Price C, Wise R, Josephs O, Frackowiak RS (1996) Functional anatomy of a common semantic system for words and pictures. Nature 383:254-256.

Van Overwalle F (2009) Social cognition and the brain: a meta-analysis. Hum Brain Mapp 30:829-858.

Vogt BA, Laureys S (2005) Posterior cingulate, precuneal and retrosplenial cortices: cytology and components of the neural network correlates of consciousness. Prog Brain Res 150:205-217.

Wagner AD, Shannon BJ, Kahn I, Buckner RL (2005) Parietal lobe contributions to episodic memory retrieval. Trends Cogn Sci 9:445-453.

Willms JL, Shapiro KA, Peelen MV, Pajtas PE, Costa A, Moo LR, Caramazza A (2011) Language-invariant verb processing regions in Spanish-English bilinguals. Neuroimage 57:251-261.

Yan C-G, Zang Y-F (2010) DPARSF: A MATLAB toolbox for "pipeline" data analysis of resting-state fMRI. Front Syst Neurosci 4:13.

Zang YF, He Y, Zhu CZ, Cao QJ, Sui MQ, Liang M, Tian LX, Jiang TZ, Wang YF (2007) Altered baseline brain activity in children with ADHD revealed by resting-state functional MRI. Brain Dev 29:83-91.

Zhang D, Raichle ME (2010) Disease and the brain's dark energy. Nat Rev Neurol 6:15-28. 